\title{
Construction of Solar-Wind-Like Magnetic Fields
}

\author{
D. Aaron Roberts \\ NASA Goddard Space Flight Center, Code 672, Greenbelt, MD 20771
}

(Dated: October 9, 2012)

\begin{abstract}
Fluctuations in the solar wind fields tend to not only have velocities and magnetic fields correlated in the sense consistent with Alfvén waves traveling from the Sun, but they also have the magnitude of the magnetic field remarkably constant despite their being broadband. This paper provides, for the first time, a method for constructing fields with nearly constant magnetic field, zero divergence, and with any specified power spectrum for the fluctuations of the components of the field. Every wave vector, $\mathbf{k}$, is associated with two polarizations; the relative phases of these can be chosen to minimize the variance of the field magnitude while retaining the "random" character of the fields. The method is applied to a case with one spatial coordinate that demonstrates good agreement with observed time series and power spectra of the magnetic field in the solar wind, as well as with the distribution of the angles of rapid changes ("discontinuities"), thus showing a deep connection between two seemingly unrelated issues. It is suggested that using this construction will lead to more realistic simulations of solar wind turbulence and of the propagation of energetic particles.
\end{abstract}

PACS numbers: 95.50.Bh, 96.50.Ry, 96.50.Tf 


\section{INTRODUCTION}

Over forty years ago, solar wind fluctuations were found to have many of the characteristics of a very basic mode of a magnetohydrodynamic plasma, namely, that of the simple transverse motion of plasma such that the field lines were "frozen in," with velocity and magnetic field fluctuations highly correlated, and such that the magnitude of the magnetic

field was essentially constant [1]. This Alfvén mode is easy to visualize as a circularly polarized, one-dimensional, monochromatic wave, but the reality is that the fluctuations are three-dimensional and broadband, consistent with a turbulent spectrum. The construction of a magnetic field with a given power spectrum and $|\mathbf{B}| \approx$ constant is further complicated by the requirement that $\nabla \cdot \mathbf{B}=0$. This problem has resisted solution until now. A descriptive approach treats the field as a "random walk on a sphere" [2]; this approach showed the natural connection between the near constancy of the field magnitude and the observed alignment of the minimum variance of the field with the mean field direction [1], but it did not offer a construction of fields with all the above constraints. There have been partial solutions for special cases (for example, [3]). This paper develops a general construction and illustrates it with a case in one spatial dimension that reproduces a number of features of observations quite well. Particularly surprising is the appearance of "discontinuities" from a procedure that works fully in a Fourier domain, and thus is nonlocal.

\section{CONSTRUCTION PROCEDURE}

The essential realization in the construction given here is that every mode in a Fourier representation of a magnetic field has two independent polarizations, and the phases of these do not affect the spectral amplitudes. The phases are what control coherence of various kinds in the time series, including the relative constancy of the field magnitude.

The total magnetic field is taken to be a Fourier sum:

$$
\mathbf{B}(\mathbf{x})=\sum_{\text {modes }} \sum_{i=1}^{2} \mathbf{B}_{\mathbf{k} i} \sin \left(\mathbf{k} \cdot \mathbf{x}+\phi_{\mathbf{k} i}\right)+\mathbf{B}_{0}
$$

where the sum on $i$ is for the two polarizations for each wave vector $\mathbf{k}$ with vector amplitude

in $k$-space $\mathbf{B}_{\mathbf{k} i}$ and with phases $\phi_{\mathbf{k} i}$. The field is defined at three-dimensional positions $\mathbf{x}$. The two polarizations may be chosen, for example, as two linear polarizations perpendicular 
to each other and to $\mathbf{k}$. The spectrum is formed by choosing the amplitudes of the modes as a function of wave vector. The mean field, $\mathbf{B}_{0}$, may be thought of as an explicit $\mathbf{k}=0$ component. Typical turbulence spectra in the solar wind are of a power law form. An isotropic case would be given by:

$$
\mathbf{B}_{\mathbf{k} i}=A k^{\alpha}
$$

with $k=|\mathbf{k}|, A$ a constant, and $\alpha$ chosen to match the spectrum of interest, e.g., $\alpha=-11 / 6$ for 3-D fields will give a Kolmogoroff $-5 / 3$ reduced spectrum as measured by a probe in the medium. The spectrum may have multiple power laws yielding, e.g., a "correlation scale" and it may be anisotropic with, e.g., "slab" and "quasi-2-D" components, as observed in the solar wind (e.g., [4], Section 3.1.3). The requirement of zero divergence is met by having $\mathbf{k} \cdot \mathbf{B}_{\mathbf{k}}=0$ for every mode. The phase changes of the current procedure do not affect the spectra of the components. It is also easy to add in velocity components, with or without Alfvénic correlations, as is done in standard MHD simulations (e.g., [5]).

The condition that $|\mathbf{B}| \approx$ constant is satisfied by an iterative procedure. Initially, one of the independent polarizations for all the modes is generated using the specified amplitudes and random phases. The other polarization is added in a mode at a time, starting with the lowest wave vectors (and thus largest amplitudes), and proceeding systematically to smaller scales. In each case, the variance of the field magnitude is calculated for a uniformly spaced set of possible phases, and the phase is chosen to minimize the variance. Thus, each step is guaranteed to lower the variation in $|\mathbf{B}|$. All wave vectors must have their phases adjusted

in this way (see below). A second optimization is the performed, replacing the randomly phased polarizations of the initial component by fixing the calculated set of phases and again minimizing the variance as modes are added in with specified amplitudes. Starting with the smallest modes did not produce a significant decrease in the variance, and performing a second iteration of the procedure, again fixing phases of one component and varying the other, give somewhat different fields, but no improvement in $|\mathbf{B}|$ constancy.

\section{RESULTS}

In one spatial dimension-equivalent to a temporal dimension for a spacecraft in the solar wind since the flow is much faster than the wave speeds-the above procedure simplifies, but still yields significant results. The divergence condition gives that the field in the direction 
of the independent coordinate (say, $x$ ) is a constant, $B_{0}$, here taken to be 1.0. The two independent polarizations can then be taken to be the $y$ and $z$ field components. The desired spectrum, in this example taken to have a spectral slope of $-5 / 3$, can be generated using random phases and spectral amplitudes given by a $-5 / 6$ power law. With $\mathrm{A}=1.0$ in the Eq. (2) above, this yields the time series shown in Fig. 1. Here, 500 points were generated using the maximum number of 250 modes. (More modes just cause aliasing.)

The one missing condition is the constancy of the field magnitude. First, the randomlyphased $y$-component of the field is held fixed, and the $z$-component is constructed starting at the lowest $k$ mode. Two hundred uniformly-spaced phases were tested for each mode, and the one that minimized the standard deviation in $|\mathbf{B}|, \delta|\mathbf{B}|$, is chosen for each mode. For this instance, $\delta|\mathbf{B}|$ decreased from 0.44 to 0.21 . For comparison, each of the components had a standard deviation of 1.02. The final step is to fix the phases that were generated for the $z$-component and regenerate the phases of the originally randomly phased $y$-component. This further reduced $\delta|\mathbf{B}|$ to 0.15 and yields the very constant appearing result seen in Fig. 2.

Figure 3 shows the result of the procedure in the Fourier space in which the series of Figs. 1 and 2, taken now to be time series, were FFTed and then log-smoothed around each frequency, $f$, such that the spectrum is averaged over $0.9 f$ to $1.1 f$. The sum of the component spectra is fixed by the construction and is not smoothed. The other two spectra are for the magnitude time series with random phases (dotted) and with the optimized phases (bottom trace). Both of these have very nearly the same slope as the component series, although this was not explicitly part of the construction.

Figure 4 presents observations of 500 1-second cadence points of solar wind magnetic field from the MFI instrument on the ACE spacecraft starting at at 15:36 UTC on 1 Oct 2007. Intervals of this duration differ considerably in their appearance, but ones like this, with a number of rapid changes interspersed with random looking fields are common. Of course the solar wind fields, unlike those of the 1-D construction, have three independent fluctuating components and a changing mean field direction, but qualitatively the agreement between Fig. 4 and Fig. 2 as compared to between Fig. 4 and Fig. 1 is striking. Even more striking is the agreement between the spectra of the measured and constructed components and magnitude, shown in Fig. 5. The constructed spectra were multiplied by a factor of 3, but otherwise were unchanged, and all but the constructed component spectrum were 
log-smoothed in the same way. Of course, there is a great deal of variability in spectra, and the agreement shown here is somewhat fortuitous, but this was the first case in which this spectral comparison was made, and not the result of extensive searching for agreement. The examination of many spectra shows that this case is fairly typical.

Note that the minimization of the field variance must be done for all the wave vectors, even though the graph of the time series of $|\mathbf{B}|$ changes almost imperceptibly when the phases are changed for the (smaller amplitude) high- $k$ components. The spectrum of $|\mathbf{B}|$ for the measured solar wind remains suppressed by about the same amount across all scales, and this is only achieved in the construction if the phase shifting is applied to all k's. Any subrange of modes that is allowed to have random phases reverts back to approximately the same power spectral amplitude as in the original, fully random-phased field. The small variance of the field magnitude occurs at all scales.

It is surprising that the method naturally generates "discontinuities" despite using a minimization method that only operates in $k$-space, and thus nonlocally. As each higher mode is added in, the time series develops steeper structures that, in an animation, almost appear as the steepening associated with the formation of shocks in a compressible-MHD simulation. The initially random component steepens similarly when it is reconstructed based on the construction of the first component. The basic conclusion is that such sharp changes are essential to the result. Figure 5, based on a 4000 point construction like that above and a (random) sample of ACE data from 10 January 2007, shows that the distribution of the angles between two field vectors separated in time by a fixed amount [9] is reproduced well by the constructed fields, but not by the random fields; the latter lack large changes. The steepening is reminiscent of the result of Cohen and Kulsrud [6] who showed that the natural asymptotic state of a 1-D compressible MHD fluid is one in which the field magnitude is constant and rapid rotations occur. The dynamics in that case equalized the magnetic field pressure across the domain. There is direct evidence in the solar wind that the fluctuations become progressively more pressure balanced [7], but this involves both the plasma and magnetic pressure, and it settles in more fully in the outer heliosphere, whereas the constancy of $|\mathbf{B}|$ implies a pressure balance of just the magnetic field, and it occurs before the $0.3 \mathrm{AU}$ closest approach of past and existing spacecraft. Thus, while it may be that compressive effects are responsible for the constancy of the field magnitude, this is far from proven, and the construction offered here does not answer the question of 
origins. There have been many approaches to the origin of discontinuities in the solar wind (for example, [8-10]); lack of space precludes further discussion here, but the present work shows a deep connection between two problems ( $|\mathbf{B}|$ variance and discontinuities) that are seemingly unrelated.

This construction also shows that the presence of sharp structures need not imply a steepening of the spectrum. Although a step or "jump" in a time series, by itself, yields a -2 spectral slope, the discontinuities imbedded in the fields here, by design, have no effect on the spectrum (cf. [11, 12]). Rapid changes imply phase coherence, but need not imply changes in the amplitude or slope of the spectrum.

The generalization of this procedure would essentially treat each direction in k-space the same was as done here for the one direction; each $\mathbf{k}$ has two polarizations, and one of them would have initial random phases. The 3-D space would then be traversed starting with low $|\mathbf{k}|$ with phases chosen to minimize the magnitude variance, etc., as above. The resulting fields could then be explored using 1-D cuts, as is often done, or be combined with velocity fields to perform MHD simulations. These are topics for future studies.

\section{DISCUSSION}

This paper has presented a means for constructing a magnetic field with zero divergence, a given power spectrum, and with a nearly constant field magnitude. The latter condition implies that the phases of the two polarizations for each wave vector are not random. The construction uses the minimization of $\delta|\mathbf{B}|$ with respect to possible phases, initially based on random phases in one of the polarizations, to produce results consistent with observations. The generalization of the construction to higher dimensions is straightforward, and should both reveal interesting comparisons with simulation results and form the basis of initial conditions for simulations. Studies of the propagation of energetic particles in the resulting fields may also help in the understanding of such issues as the observed power spectrum of the particles; recent work with ad hoc intermittent turbulence [13] is illustrative of potential effects, although the details there are quite different. The minimum variance results seen in the solar wind have been difficult to reproduce in simulations (e.g., [14] wherein the anisotropies found are much smaller than those in the solar wind), and it is possible that the difficulty is the lack of the near constancy of the field in the initial conditions. Generalizing 
this method to an expanding geometry will be more challenging, although it can be applied to "expanding box" simulations [15] in the present form.

A number of qualitative features of the results are notable. First, the relative constancy of the magnetic field fluctuations occurs at all scales; it is not simply that the variance is small in a global sense. Although the construction here assumes a mean field, $\mathbf{B}_{\mathbf{0}}$, the arguments by Barnes [2] imply that the fields constructed here will, even in 3-D, be nearly perpendicular to a local mean field in addition to showing a total minimum variance along the global mean field. Next, the appearance of "discontinuities" in the time series seems to be required by the near constancy of the magnitude. Nothing about the method was predisposed to making rapid changes in the fields, but they frequently arise nonetheless. This "patchy" behavior is reminiscent of nonlinear dynamical evolution in MHD [16], which also may be relevant to the creation of the states seen here. Note also that the rapid field changes arose despite the fixed spectral shape; there was no steepening or other change in the spectrum required to make them, only a degree of coherence in the phases. There are other sorts of fine scale structures, such as "pulsed Alfvén waves" [17], that also occur in the fields constructed here (see the solid-line-component dips near points 400 and 440 of Fig. 2); their appearance in this general construction supports the idea that these structures are intrinsic to a turbulent magnetofluid.

It still remains a puzzle how the near constancy of $|\mathbf{B}|$ arises. It is observed as close to the Sun as we have in situ measurements, so it must occur early in the wind evolution. Compressive effects that can steepen structures also tend to remove variability in the magnitude of the field [6], and thus these could be at work, but as yet we do not have clear proof. Kinetic effects may also play a role, but again this remains to be shown.

The author thanks the ACE MFI team and NASA's Space Physics Data Facility for provision of data, and NASA's LWS TR\&T program for support of this research.

[1] J. W. Belcher and L. Davis, Jr., J. Geophys. Res. 76, 3534 (1971).

[2] A. Barnes, J. Geophys. Res. 86, 7498 (1981).

[3] B. J. Vasquez and J. V. Hollweg, J. Geophys. Res. 103, 335 (1998).

[4] R. Bruno and V. Carbone, Living Reviews in Solar Physics 2, 4 (2005). 
[5] T. Stribling, D. A. Roberts, and M. L. Goldstein, J. Geophys. Res. 101, 27603 (1996).

[6] R. H. Cohen and R. M. Kulsrud, Physics of Fluids 17, 2215 (1974).

[7] D. A. Roberts, J. Geophys. Res. 95, 1087 (1990).

[8] A. Greco, W. H. Matthaeus, R. D’Amicis, S. Servidio, and P. Dmitruk, Astrophys. J. 749, 105 (2012).

[9] J. E. Borovsky, Journal of Geophysical Research (Space Physics) 113, A08110 (2008).

[10] M. Neugebauer and J. Giacalone, Twelfth International Solar Wind Conference 1216, 194 (2010).

[11] G. L. Siscoe, L. Davis, Jr., P. J. Coleman, Jr., E. J. Smith, and D. E. Jones, J. Geophys. Res. 73, 61 (1968).

[12] J. E. Borovsky, Physical Review Letters 105, 111102 (2010).

[13] M. Zhang and R. Schlickeiser, Astrophys. J. 756, 129 (2012).

[14] W. H. Matthaeus, S. Ghosh, S. Oughton, and D. A. Roberts, J. Geophys. Res. 101, 7619 (1996).

[15] R. Grappin and M. Velli, J. Geophys. Res. 101, 425 (1996).

[16] S. Servidio, W. H. Matthaeus, and P. Dmitruk, Physical Review Letters 100, 095005 (2008).

[17] J. T. Gosling, H. Tian, and T. D. Phan, Apstrophys. J. Lett. 737, L35 (2011). 


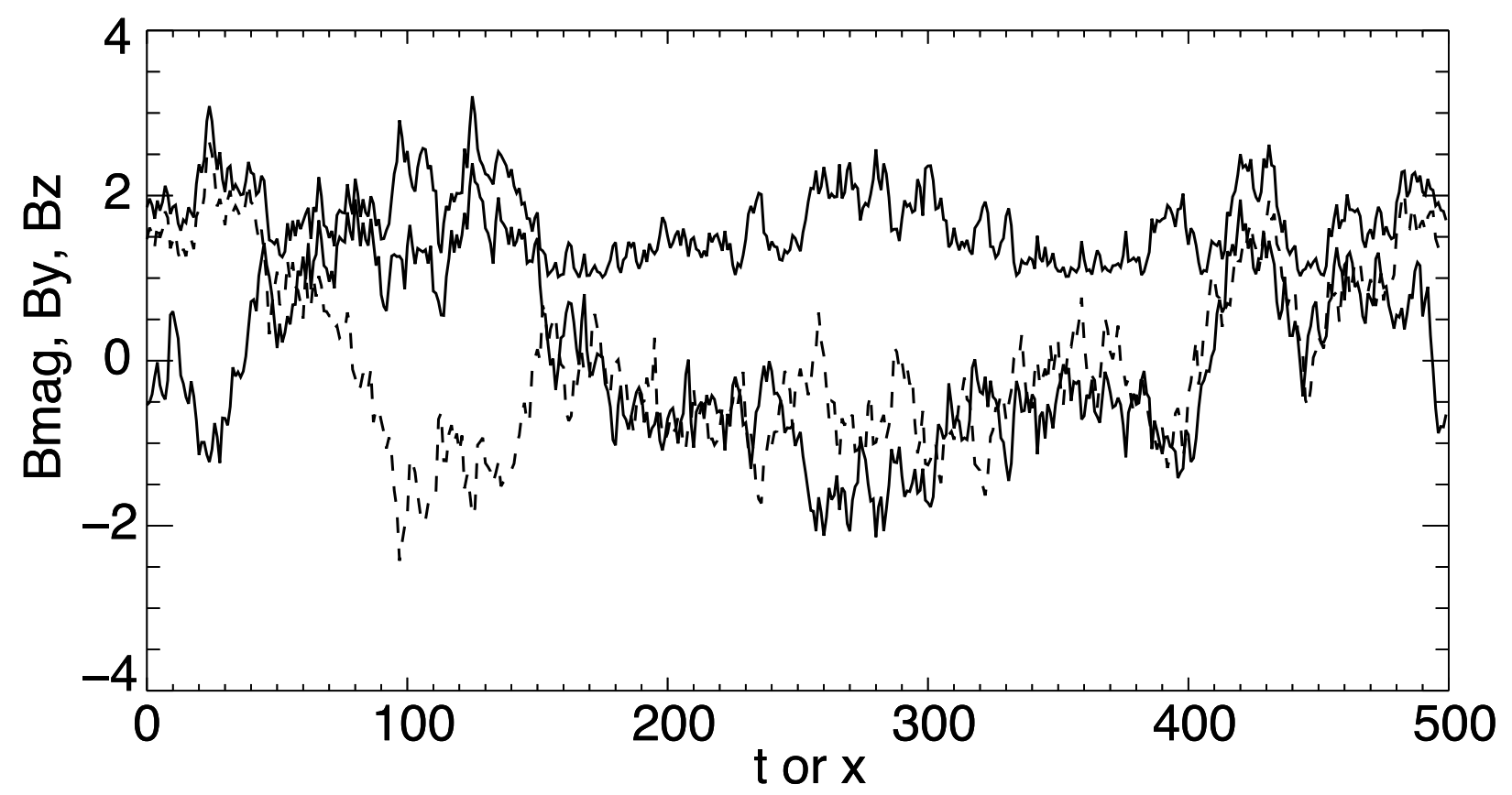

FIG. 1. Time series of artificially generated magnetic fields for random phases that depend on one dimension. The top trace is the field magnitude, and the other two traces are the components of the transverse fields.

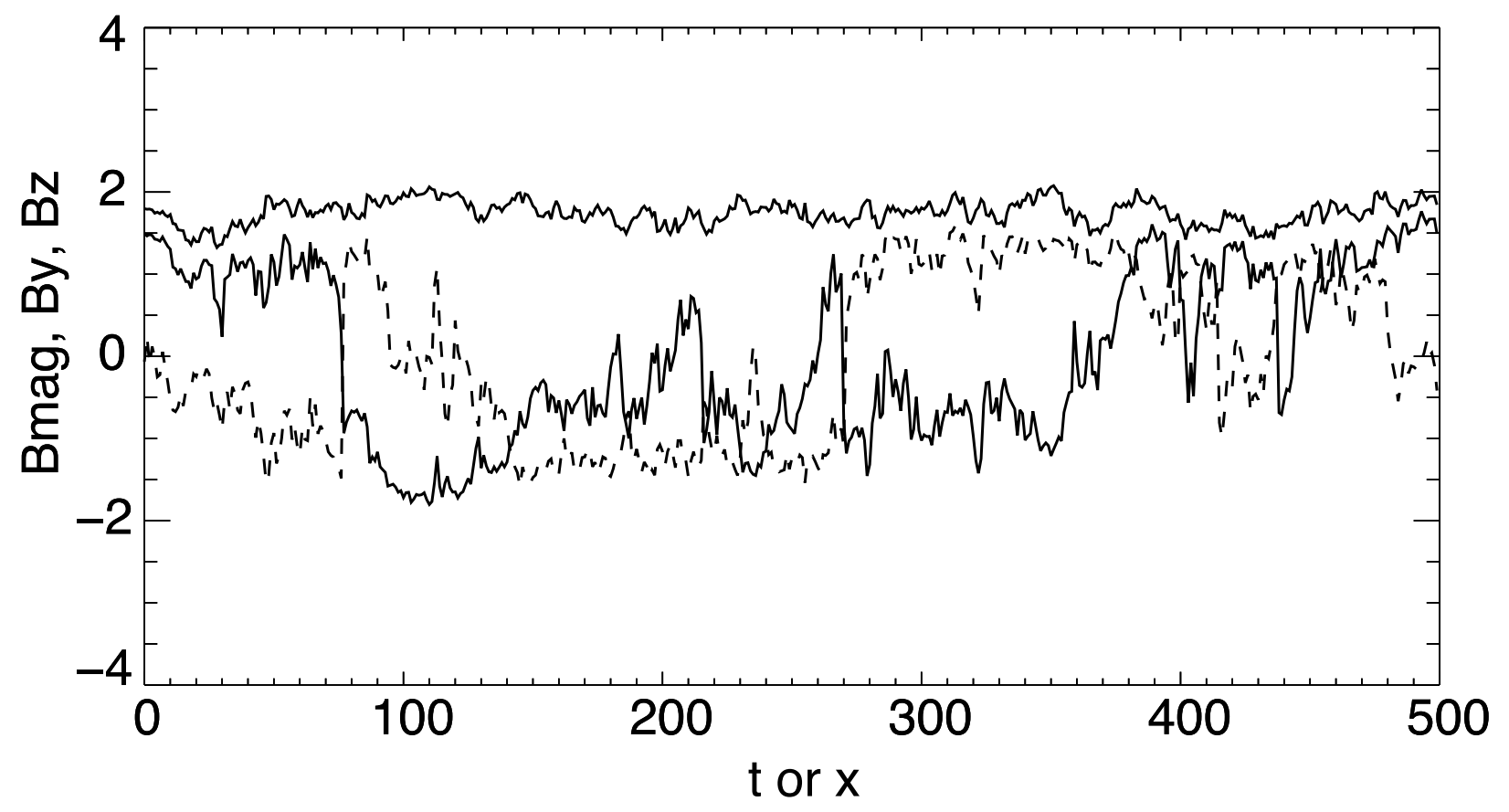

FIG. 2. Time series of artificially generated magnetic fields after the phase optimization. Note that the magnitude of the field is now quite constant. 


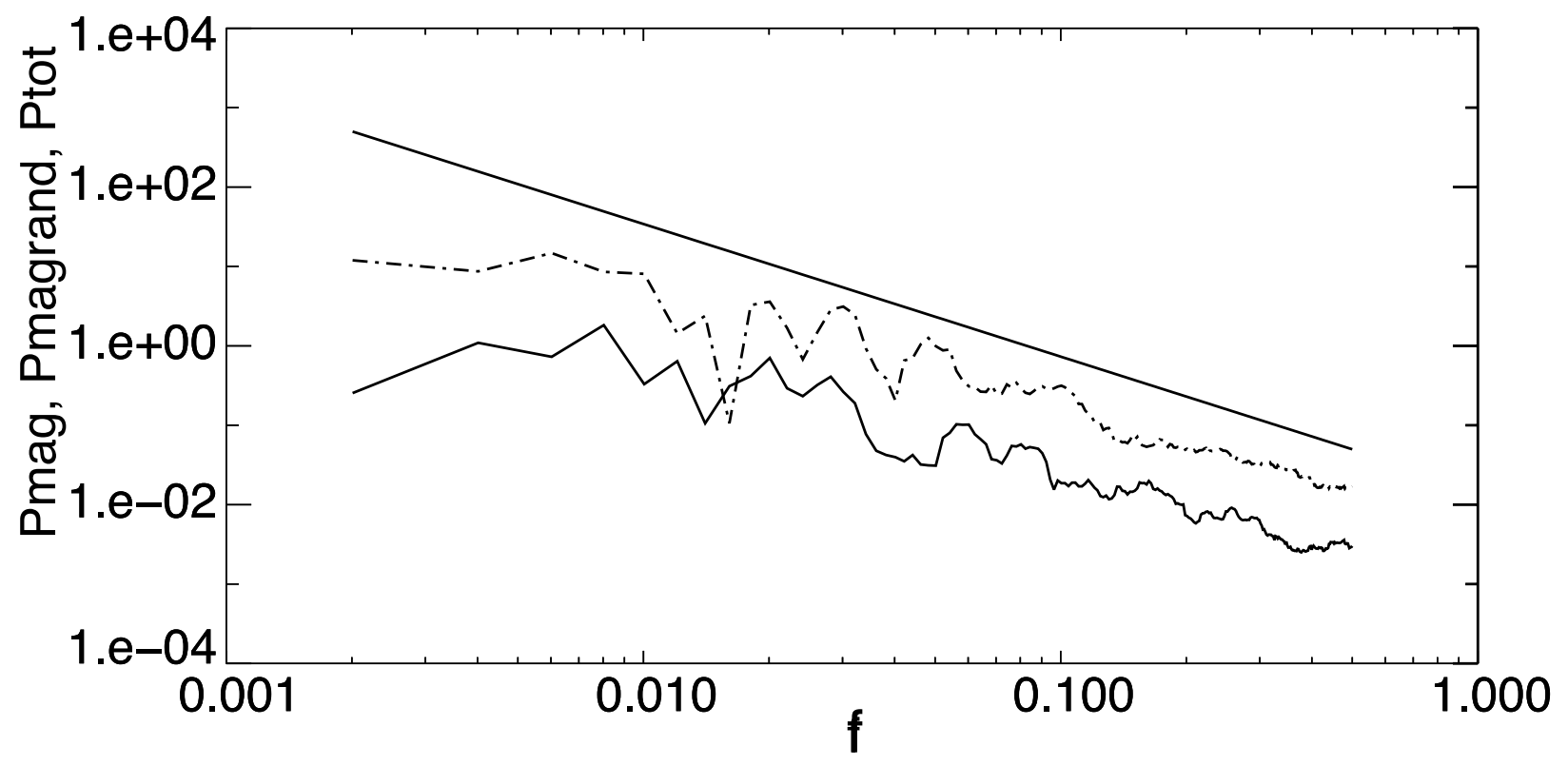

FIG. 3. Spectrum of artificially generated magnetic fields. The upper line is the total power in the transverse components, the dashed line is the power in the magnitude of the field for random phases, and the lowest line is the power in the magnitude after the optimization of the phases.

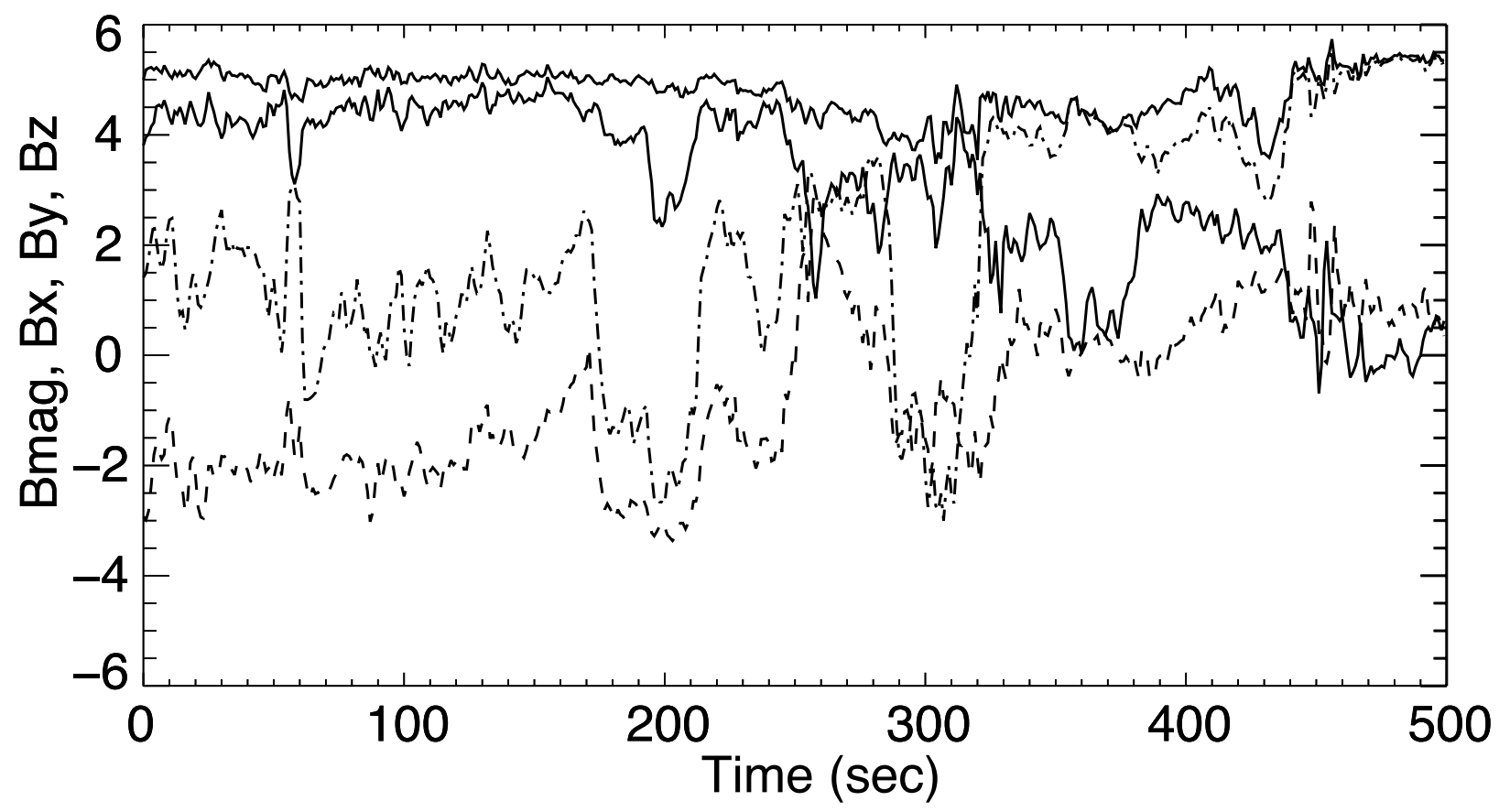

FIG. 4. Time series of measured magnetic fields. Data are 1-second ACE magnetometer data starting at 1 October 2007, 15:36 UTC. The top trace is $|\mathbf{B}|$, and the other traces are for the components: radial (solid), tangential (dot-dashed), and normal (dashed). 


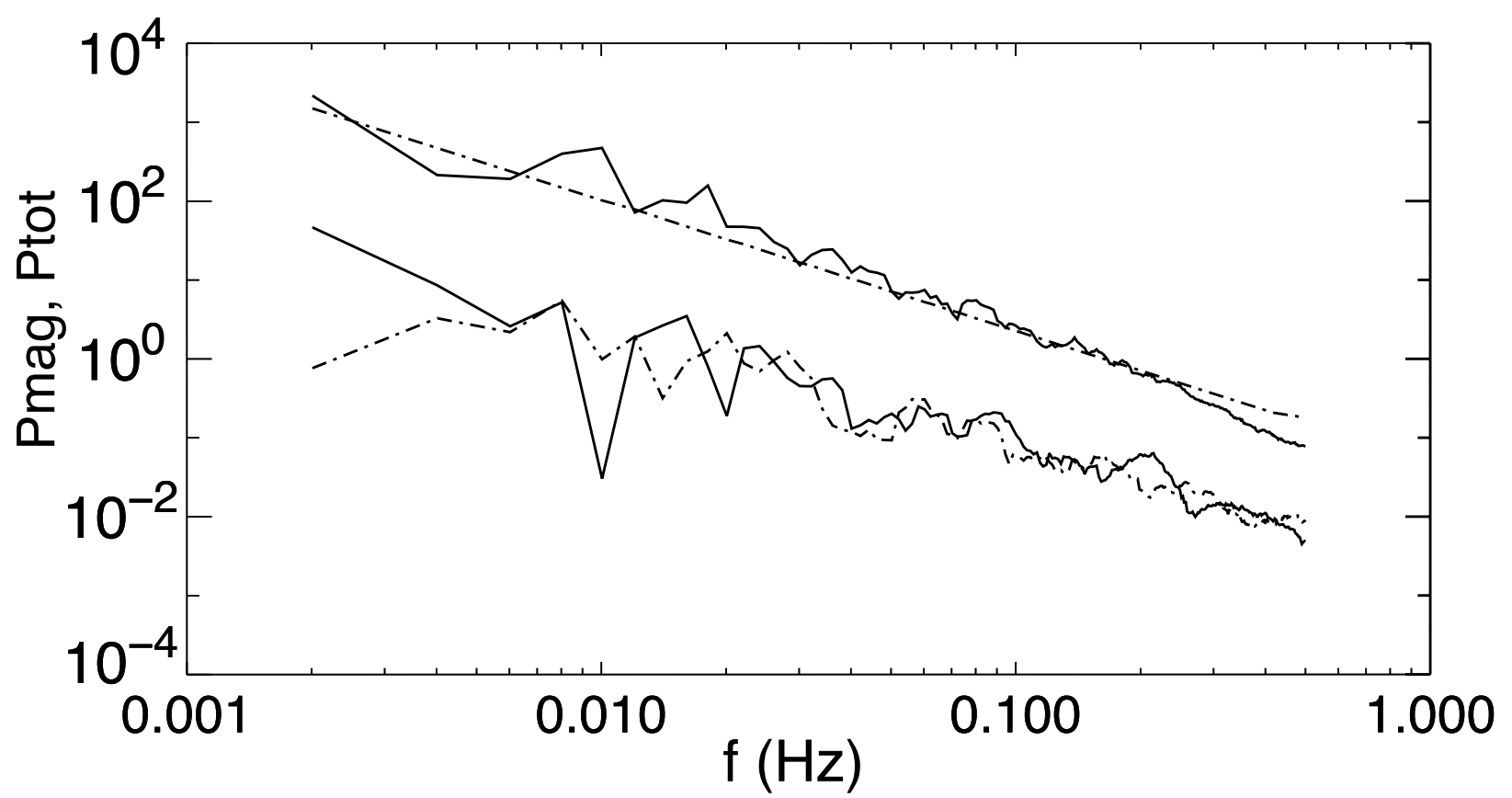

FIG. 5. Spectrum of measured magnetic fields (solid; sum of component spectra above, and power in the magnitude below) and of the constructed fields (dot-dashed). Units for the measured spectrum are $n T^{2} / H z$.

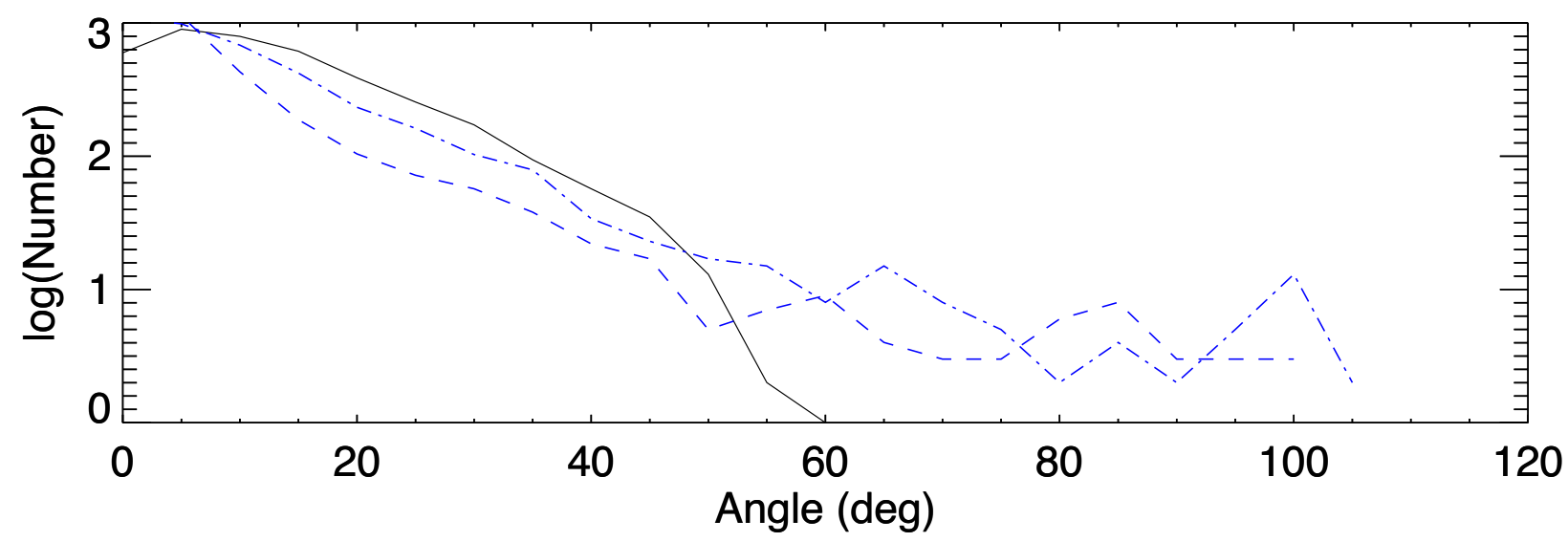

FIG. 6. Distribution of angles between 3920 vectors measured 15 points apart. The solid line is for the randomly phased construction, the dashed line for the optimized phase case, and dot-dashed line is the ACE result. 